\title{
Provable Correctness of Prototype Interpreters in LDL
}

\author{
Ralf Lämmel, Günter Riedewald \\ Universität Rostock, Fachbereich Informatik \\ 18051 Rostock, Germany \\ E-mail: ( rlaemmel I gri )@informatik.uni-rostock.de
}

\begin{abstract}
LDL is a system supporting the design of procedural programming languages and generating prototype interpreters directly from the language definition. A language definition is based on GSFs - a kind of attribute grammars - and the denotational semantics approach. It is shown how denotational semantics can be transformed into Prolog. It is also shown how the correctness of the prototype interpreters can be proved using a technique developed by Clark and extended by Deransart.
\end{abstract}

\section{Introduction}

A particularly important problem in software development is to guarantee the functional correctness of software. Because the theory of programming languages and their implementation is well-understood and there is a close relation between theory and practice, compilers and interpreters could be the first complex software systems developed in a manner guaranteeing correct products by automatizing as much steps as possible and by using suitable correct tools including preproduced correct components.

But what does it mean "a compiler (including interpreter) is correct" ? According to [KPy80], [Sch76] a translation is correct iff it is semantic-preserving, i.e. a source program and its corresponding target program have the same meaning. [KPy80] gives some minimal set of sufficient conditions guaranteeing the property of semantic-preserving and exploits a generalization of attribute grammars to define semantics, whereas in [Sch76] an algebraic approach is used where language constructs are elements of a typed word algebra. [TWW81], [M73] apply an algebraic approach based on syntactical algebras. Boot-strapping techniques as used in [PRO92] or in [To90] can support the development of correct compiler generators.

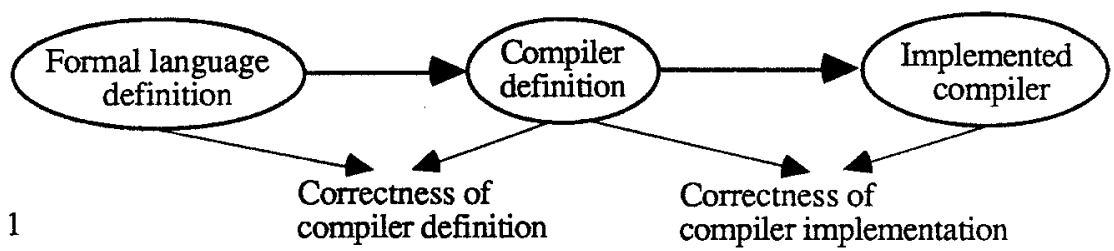

To define compiler (including interpreter) correctness we will start with the language definition (Fig. 1). A formal language definition is the fundamental of a compiler definition. According to the compiler definition the compiler must be implemented. The question of correctness appears at the following points of the whole process:

- The language definition and the compiler definition define the same language (correctness of compiler definition): For any program $\mathrm{p}$ its input-output behaviour w.r.t. the language definition is identical with the input-output behaviour of the compiled program p w.r.t. the compiler definition. 
- The compiler definition and the implemented compiler define the same language (correctness of compiler implementation): For any program p once compiled w.r.t. the compiler definition and once compiled using the implemented compiler the input-output behaviour is the same.

The concept used in our project LDL ([R91a], [R91b], [R92], [Lä93], [RL93]) is aimed at the development of correct prototype interpreters directly from the language definition thereby mixing both mentioned notions of compiler correctness. Our language definitions consist of

- a GSF (Grammar of Syntactical Functions - a kind of attribute grammars) describing the concrete syntax and the static semantics,

- a denotational semantics definition describing the dynamic semantics.

The derived prototype interpreters are written in Prolog. This language was selected, because:

- GSFs are closely related to Prolog programs and after some modifications a GSF can directly be used as a Prolog program offering the core of a prototype interpreter.

- If the syntax of the language can be defined by an LL(k)-grammar there is no need of a parser because the Prolog system itself can be exploited.

- It is well-known that in general in Prolog programs the same parameter positions can be used once for input and once for output if certain conditions are satisfied. In LDL this property is exploited to derive test program generators from the same language definition as it is used for prototype interpreters.

- The correctness of the prototype interpreter, especially of the implemented denotational semantics definition, w.r.t. the language definition can be proved applying the inductive proof method ([D90]).

- Other parts of $L D L$, that is to say the knowledge base and the tool for language design (see Section 2), are preferably to be implemented in a logic programming language. Therefore the fact that the prototype interpreters are implemented in Prolog contributes to the technical simplicity of LDL.

The rest of the paper is subdivided as follows. Section 2 describes the basic concepts of the LDL system. In Section 3 basic notions are mentioned. Section 4 describes our approach to correct prototype interpreters.

\section{The LDL concept}

\subsection{Structure of LDL}

Keeping in mind Koskimies' statement ([K91]) "The concept of an attribute grammar is too primitive to be nothing but a basic framework, the 'machine language' of language implementation." LDL offers a higher-level tool supporting the definition of procedural languages and their implementation in form of a prototype interpreter. For this purpose the LDL library contains predefined language constructs together with their denotational meaning expressed by Prolog clauses. These semantics components are correct w.r.t. the usual denotational definition (see Section 4). The knowledge base and the tool for language design ensure the derivation of correct prototype interpreters from these correct components. At present the library contains all usual Pascal constructs and some additional constructs. 
The system LDL is based on the idea from [R91a] and exploits GSFs for the language definition. Moreover, in LDL there is a test program generator producing syntactically correct programs satisfying the context conditions of the defined language and possessing certain additional properties. For more exhaustive considerations of LDL and provable correct prototype interpreters within LDL the reader is refered to [RL93].

\subsection{Prototype interpreters}

The structure of a prototype interpreter can be seen from Fig. 2. The development of prototype interpreters is based on the following ideas:

- Because GSFs and Prolog programs are closely related a source language definition in form of a GSF may be the core of a prototype interpreter written in Prolog. The syntactical and semantical structure of the language is described by the GSF.

- The semantics definition can be a stepwise process. First, we could be interested only in the calling structure of the semantic functions of a given source program. Finally, we are interested in the computation of the source program. Thus our semantics definition consists of two levels:

1. The meaning of a program is a term consisting of names of semantic functions in the GSF sense which can be considered as the abstract syntactical structure of the program. It can be defined using a GSF with special production rule patterns.

2. Based on the denotational approach the interpretation of terms is defined by Prolog clauses.

- Before computing the meaning of a source program its context conditions are checked.

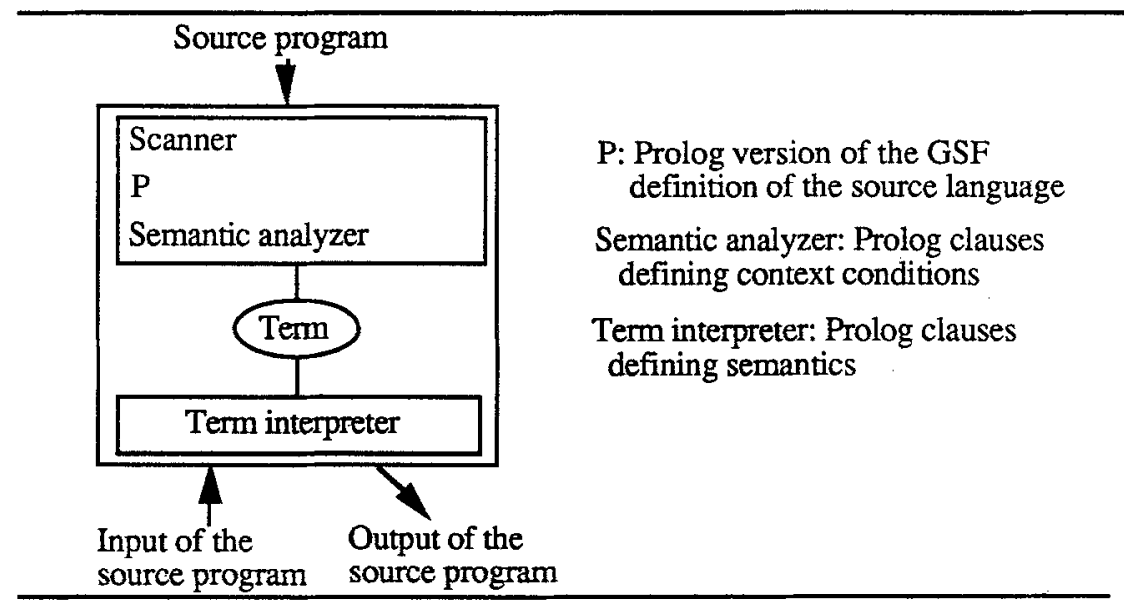

Fig. 2

The prototype interpreter operates as follows:

- A source program is read token by token from a text file.

- Each token is classified by a standard scanner. It is a part of LDL.

- The parsing and checking of context conditions is interconnected with scanning. If the context-free basic grammar of the GSF describing the source language is an LL(k)grammar the Prolog system itself can be used straightforwardly for parsing, whereas LR(k)-grammars require to include a special parser. 
- Recognizing a language construct its meaning in form of a term is constructed by connecting the meaning of its subconstructs.

- The term representing the meaning of the whole program is interpreted by associating the function names of the term with functions which e.g. transform program states.

\section{Basic definitions}

\subsection{Denotational semantics}

Our semantics descriptions are recursive function definitions describing the mapping of syntactical elements to their meanings. Syntactical domains are considered as sets of terms, whereas semantic domains are assumed to be complete partial orders (cpos). The reader is expected to be familiar with conventional denotational semantics ([St77]). Here, we will mention only some basic functions and notational conventions applied in this paper.

Let be BOOLEAN $=\{\text { true,false }\}_{\perp}, \mathrm{D}, \mathrm{D}_{1}, \ldots, \mathrm{D}_{\mathrm{n}}$ arbitrary semantic domains (cpos). The following basic functions are assumed to be available:

- projections eli for product domains $\left(D_{1} \times \ldots \times D_{n}\right)$, the usual list operations head, tail, cons, null for domains of sequences $D^{*}$, operations out (mapping from sum to the $i$-th summand), $\underline{i_{i}}$ (vice versa), $\underline{i s}$ or $\underline{i s} D_{i}$ (test predicate for the $i$-th summand) for sum domains $\left(D_{1}+\ldots+D_{n}\right)$, equal as usual predicate for equality for any $D$

- cond: BOOLEAN $\times \mathrm{D} \times \mathrm{D} \rightarrow \mathrm{D}$ to express conditionals (the arrow notation $\mathrm{b} \rightarrow \mathrm{d}_{1}, \mathrm{~d}_{2}$ of cond $\left(\mathrm{b}, \mathrm{d}_{1}, \mathrm{~d}_{2}\right)$ is sometimes prefered)

- functional operator for error propagation:

$\ldots \otimes \ldots:\left(\mathrm{D}+\{\text { error }\}_{\perp}\right) \times\left(\mathrm{D}->\left(\mathrm{D}+\{\text { error }\}_{\perp}\right)\right) \rightarrow\left(\mathrm{D}+\{\text { error }\}_{\perp}\right)$

$\mathrm{d} \otimes \mathrm{f}=\{$ error, if $\mathrm{d}=$ error

$\{\mathrm{f}($ out $1(\mathrm{~d}))$, else

- modification operator ... [ . . / . . ], where $f\left[y_{0} / x_{0}\right]$ returns a function $f^{\prime}$ with $\mathrm{f}^{\prime}\left(\mathrm{x}_{0}\right)=\mathrm{y}_{0}$ and $\forall \mathrm{x} \neq \mathrm{x}_{0}: \mathrm{f}^{\prime}(\mathrm{x})=\mathrm{f}(\mathrm{x})$

Elements of product domains and domains of sequences are enclosed in brackets $<$ and $>$. Elements of sum domains are represented as pairs (d,i), where i refers to the summand of the operand which the element $d$ was taken from. For sum domains we apply as usual some kind of simple unification, i.e. $\exp 1=x->\exp 2, \exp 3$ (where $x$ is meta-variable of summand domain $\left.\mathrm{D}_{\mathrm{i}}\right)$ is an abbreviation for $\underline{\underline{i}} \mathrm{i}(\exp 1) \rightarrow(\lambda x \cdot \exp 2)$ out ${ }_{i}\left(\exp _{1}\right)$, exp3.

\subsection{Logic programming and correctness}

The reader is expected to be familiar with the basic notions of logic programming. Here we shall only include the main definitions needed for the inductive proof method ([D90]).

Let $S$ be a set of sorts, $F$ an $S$-signature (declaring the signature of function symbols over $\mathrm{S}), \mathrm{V}$ an $\mathrm{S}$-sorted set of variables. The free $F$-algebra $\mathrm{T}(\mathrm{F}, \mathrm{V})$ is identified as the set of the well-formed and "well-typed" terms. The term algebra T(F) is identified with the set of all ground terms. For both algebras $T(F, V)$ and $T(F)$ the operations are interpreted as usual as term constructors. $T(F, V)_{S}\left(T(F)_{S}\right)$ denotes the set of all (ground) terms of sort $s$. 
To write down specifications for logic programs describing the relations which should be defined by the clauses of a logic program we will use a logical language (first order) with a specific interpretation. A logical language $L$ is a 4-tupel $\langle S, F, R, V\rangle$, where $S$ is a set of sorts containing the sort bool interpreted as $B O O L=\{$ true,false $\}, F$ an $S$-signature, $R$ a set of many-sorted predicate symbols (every $\mathrm{p} \in \mathrm{R}$ is of sort bool) and $\mathrm{V}$ an $S$-sorted set of variables. $L$ contains all formulae over $\mathrm{V}, \mathrm{F}, \mathrm{R}$ and applying connectives $\forall, \exists,<=>$, $\Rightarrow$, $\wedge, v, \sim$. By $\phi\left[t_{1} / v_{1}, \ldots, t_{n} / v_{n}\right]$ the result of the substitution of variables $v_{i}$ by terms $t_{i}$ in the formula $\phi$ is denoted. We denote by free $(\phi)$ the possibly empty set of the free variables of the formula $\phi$ of $L$.

To interprete formulae from $L$ we need a so-called $L$-structure which declares the interpretation of the function and predicate symbols. An $L$-structure $\mathbf{D}$ is a structure of the form $<\left\{D_{S}\right\}_{S} \in S,\{f D\}_{f} \in F,\{\mathbb{D}\}_{r \in R}>$, with $S, F, R$ defined as above and where $<\left\{D_{S}\right\}_{S} \in S,\left\{f_{D}\right\}_{f} \in F>$ is a heterogeneous F-algebra and for each $r$ in $R$ rD is a total mapping $\mathrm{TD}: \mathrm{D}_{\mathrm{S} 1} \times \ldots \times \mathrm{D}_{\mathrm{sn}} \rightarrow \mathrm{BOOL}$. A formula $\phi$ in $\mathrm{L}$ is valid in $\mathbf{D}(l=\mathbb{D} \phi)$, iff for all assignments $v$ the interpretation of $\phi$ yields true.

In this paper logic programs are written down in Prolog notation (Edinburgh syntax), although we restrict ourselfs (for most parts) to definite clause programs (DCPs) neglecting their evaluation by a particular Prolog system. A DCP P is a 4-tupel <PRED,FUNC,CLAUS,VAR>, where PRED is a finite set of predicate symbols, FUNC a finite set of function symbols, FUNC $\cap$ PRED $=\varnothing$, VAR some set of variables, CLAUS a finite set of clauses defined as usual over PRED and TERM $=T$ (FUNC,VAR). $D E N(P)$ denotes the set of all atomic logic consequences of $P$.

Definition 1 (Specification $\boldsymbol{S}$ of $\mathrm{P}$ on $\mathrm{L}$ and $\mathrm{D})$ :

Let be $\mathrm{L}=\langle\mathrm{S}, \mathrm{F}, \mathrm{R}, \mathrm{V}\rangle$ a logical language, $\mathrm{D}$ an L-structure, $\mathrm{P}=\angle \mathrm{PRED}, \mathrm{FUNC}, \mathrm{CLAUS}, \mathrm{VAR}>$ a DCP. A specification $\mathbf{S}$ of $\mathrm{P}$ on $\mathrm{L}$ and $\mathrm{D}$ is a family of formulae $\mathcal{S}=\{S P\}_{p} \in P R E D$ such that $S P \in L$, VAR $\underline{\mathrm{C}} \mathrm{V}$, FUNC $\subseteq$ F. For every $p$ of PRED of arity $n, \operatorname{varg}(p)=\left\{p_{1}, \ldots, p_{n}\right\}$ is the set of variable names denoting any possible

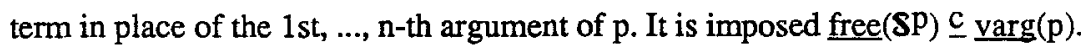

Definition 2 (Valid specification $S$ of $P$ ):

A specification $\mathbf{S}$ of $\mathrm{P}$ on $\mathrm{L}$ and $\mathrm{D}$ is valid (or $\mathrm{P}$ is correct w.r.t. $\mathbf{S}$ ) iff

$$
\forall \mathrm{p}\left(\mathrm{t}_{1}, \ldots, \mathrm{t}_{\mathrm{n}}\right) \in \mathrm{DEN}(\mathrm{P}) \mathrm{l}=\mathrm{D} \mathrm{SP}\left[\mathrm{t} 1 / \mathrm{p} 1, \ldots, \mathrm{t}_{\mathrm{n}} / \mathrm{p}_{\mathrm{n}}\right] \text {, }
$$

where $n$ is the arity of $p$. The notation will be abbreviated by $S P\left[t_{1}, \ldots, t_{n}\right]$.

Definition 3 (Inductive specification $\mathbf{S}$ for DCP P):

A specification $S$ of $P$ on $L$ and $D$ is inductive iff for every $c$ in CLAUS, c: $\mathrm{ro}\left(\mathrm{t} 01, \ldots, \mathrm{t} 0 \mathrm{n}_{0}\right):-\mathrm{r}_{1}(\mathrm{t} 11, \ldots, \mathrm{t} 1 \mathrm{n} 1), \ldots, \mathrm{rm}_{\mathrm{m}}\left(\mathrm{tm} 1, \ldots, \mathrm{tmn}_{\mathrm{m}}\right)$,

I=D $\boldsymbol{S}^{\mathrm{r}} 1[\mathrm{t} 11, \ldots, \mathrm{t} \ln 1] \wedge \ldots \wedge \boldsymbol{s}^{\mathrm{r}} \mathrm{m}\left[\mathrm{tm} 1, \ldots, \mathrm{tmn}_{\mathrm{m}}\right]$ $\Rightarrow \mathrm{S}^{\mathrm{r}} \mathrm{O}\left[\mathrm{t} 01, \ldots, \mathrm{t} 0 \mathrm{n}_{\mathrm{O}}\right]$

Proposition:

If $\boldsymbol{S}$ of $\mathrm{P}$ is inductive then $\boldsymbol{S}$ is valid for $P$. 


\section{Correctness of prototype interpreters}

\subsection{Overview}

Our aim is to prove the correctness of prototype interpreters constructed as described in subsection 2.2. As we have seen the meaning of a language construct is defined in two steps: first a term is constructed which can be considered as the abstract syntactical structure of the language construct, and second the term is interpreted by Prolog clauses. Because the term construction is described by a GSF (language definition), which then is transformed into a Prolog program, to prove the correctness of a prototype interpreter means to prove first the correctness of the GSF including its transformation and second the correctness of the Prolog clauses interpreting terms. Using a special form of production rule patterns of a GSF (see [RL93]) the first proof is trivial. Therefore, in the sequel we will focus only on the second proof.

There are two approaches how to realize this proof. The first one is an algebraic approach (see [RL93]). Here we will use another approach exploiting the inductive proof method for logic programs as described in [D90]. The idea of the method is the following. To prove the correctness of a logic program three kinds of semantics must be taken into consideration:

- The intended semantics: It describes the intentions of a programmer, e.g. in form of relations.

- The specified semantics: It describes the semantics really specified by the logic program. It can be identified with the set of all proof-tree roots of the program.

- The operational semantics: It takes into account the implementation of a logic program (computation of proof-trees).

To prove the correctness of a logic program means to prove that the intended and the specified semantics are equal and that the operational semantics "preserves as much as possible" the same semantics. In the following subsections we will focus our attention on the first problem neglecting the second one.

In our case the intended semantics will be the denotational semantics SD of a programming language and the specified semantics is given by the logic program SP intended to implement SD. Before transforming SD into SP and proving (in interconnection) the correctness of SP w.r.t. SD some modifications of SD are necessary. For clarity we will apply a simple imperative programming language VSPL (Very Simple Programming Language) as a running example. VSPL contains some simple language constructs of Pascal, namely:

- declarations of variables of type integer and boolean

- expressions built from constants and variables applying the usual arithmetic and relational operations on the domains of integer and boolean values

- empty statements, assignments, sequences, alternatives, iterations.

The general task to derive a logic program from a recursive function definition (see subsections $4.2,4.3,4.4$ ) consists of the definition of the following representations:

- Representations of elements from domains: Elements of syntactical and non-functional semantic domains, are represented by ground terms. For functional domains we give transformation rules eliminating the necessity of these domains already within the 
semantics description. This approach is sufficient at least for language descriptions of imperative languages we have considered (Pascal-like languages).

Representations of functional equations: In general, named functions defined by equations are represented within a logic program as predicates. An equation is represented by a definite clause. The intention is that a logic program applied to the representation of a function argument "computes" an answer iff the corresponding function application returns a value and the computed answer is the representation of the function result (see subsection 4.4). Therefore parameter positions of the derived predicates are divided into input and output parameter positions.

\subsection{Semantics description}

First, the syntactical domains of the considered language will be defined in an algebraic way. Therefore a set SSyn of sorts, an SSyn-signature FSyn and an SSyn-sorted set VSyn of variables will be introduced. The sorts from SSyn correspond to the final syntactical domains which are sets of terms (e.g. the syntactical domain of expressions is identified with $\mathrm{T}(\mathrm{FSyn})$ expression). A term can be considered as a linear representation of the abstract syntactical structure of a language construct. FSyn describes function symbols which can be applied to construct syntactical elements. VSyn introduces meta-variables for the semantics description. Second, semantic domains and semantic functions will be defined.

Example 1 (Excerpt of the description of VSPL):

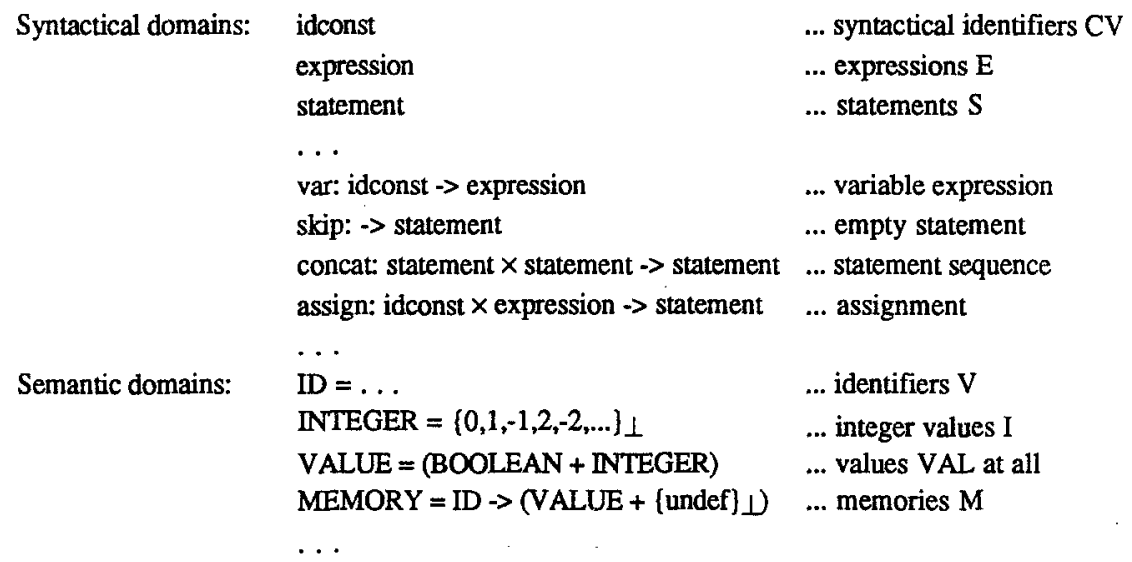

Semantic functions BDCV, IE and IC describing meanings of resp. syntactical identifiers, expressions and statements:

BDCV: $T($ FSyn) idconst $\rightarrow$ ID

IE: T(FSyn)expression $\rightarrow>$ MEMORY $\rightarrow$ (VALUE $\left.+\{\text { error }\}_{\perp}\right)$

IC: $\mathrm{T}(\mathrm{FSyn})$ statement $\rightarrow$ MEMORY $\rightarrow\left(\right.$ MEMORY $\left.+\{\text { error }\}_{\perp}\right)$

$(\mathrm{SD} .1) \mathrm{IE}(\operatorname{var}(\mathrm{CV}))(\mathrm{M})=\mathrm{M}(\mathrm{BDCV}(\mathrm{CV}))=\mathrm{VAL} \rightarrow \mathrm{VAL}$, error

(SD.2) IC (skip) (M) = $M$

(SD.3) IC (concat(S1,S2)) (M) $=\quad$ IC (S1) (M) $\otimes \lambda M^{\prime} \cdot I C$ (S2) (M')

(SD.4) IC (assign(CV,E)) (M) $=\quad$ IE (E) (M) $\otimes \lambda$ VAL.M[VAL/BDCV(CV)] 


\subsection{Transformation of the semantics description}

The derivation of the logic program SP from the denotational semantics SD requires some modifications of SD. Especially functional domains must be flattened.

\section{Representing functions by sequences}

The fact that finite functions can be considered as finite sequences of ordered pairs may be exploited to avoid some functional domains, e.g. a function $f: D_{1} \rightarrow\left(D_{2}+D^{\prime}\right)$, where $D_{1}$, $D_{2}$ are arbitrary (non-functional) semantic domains and $D^{\prime}=\{$ undef $\} \perp$ and $f$ maps nearly all $d \in D_{1}$ to undef, can be represented as an element $f^{\prime}$ of $\left(D_{1} \times D_{2}\right)^{*}$ consisting of all

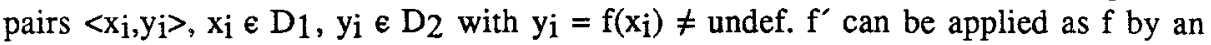
auxiliary function (see Example 2). The important substitution operator (at least for such functions) can also be redefined very easy.

Example 2 (Representing memories of VSPL by sequences):

In SD of VSPL elements of MEMORY are either the uninitialized memory or they are obtained by the application of the substitution operator. Now MEMORY is defined by MEMORY $=(\text { ID } \times \text { VALUE })^{*}$. The operators for application and substitution

$$
\begin{aligned}
& \text { APPLY: }(\mathbb{D} \times \text { VALUE }) * \times \mathbb{D} \rightarrow\left(\text { VALUE }+\{\text { undef }\}_{\perp}\right) \\
& \text { UPDATE: (ID } \times \text { VALUE) } * \text { ID } \times \text { VALUE }->(I D \times V A L U E) *
\end{aligned}
$$

can be defined following the style of recursive function definition, e.g. for APPLY:

$\operatorname{APPLY}(\mathrm{M}, \mathrm{V})=\underline{\text { null }}(\mathrm{M})->$ undef,

$$
\text { el 1 (head(M)) equal V } \rightarrow \text { el2 (head(M)),APPLY(tail(M),V) }
$$

Because of this new definition of MEMORY, modifications within SD are necessary, e.g. the right hand side of (SD.1) would become to:

$$
\text { APPLY(M,BDCV(CV)) }=\text { VAL } \rightarrow \text { VAL, error }
$$

\section{Freezing computation of meanings}

If we include e.g. procedures, semantic functions work over functional domains modelling meanings of program fragments. The environment must hold meanings of procedures beeing functional entities. To eliminate such functional domains we can exploite that concrete functions of such domains are usually only instantiated by the application of some semantic function to a syntactical object. Therefore it is possible to copy this syntactical element through the semantics description instead of its meaning (obtained by the application of the semantic function to this syntactical element) and to apply the corresponding semantic function if all arguments are known. E.g. for the procedure construct, the syntactical term beeing a procedure declaration can be bound in the environment. When the procedure is called, this syntactical term can be interpreted, since the actual parameters and the state are known. This approach was successfully applied for the implementation of the language constructs procedures, functions, escapes (exits) and gotos in a Pascal-like language. Especially continuation semantics can be implemented applying this approach.

\section{Eliminating higher-order operators}

Denotational descriptions apply generic operators with a signature often based on functional domains (e.g. the introduced $\otimes$ ) or on domains not further restricted. Such operators are intended to shorten descriptions by figuring out often used parts of 
descriptions. Functional domains arising from such operators can often be eliminated by substituting the application of the operator by the application of the definition of the operator itself (e.g. the right hand side of (SD.3) applying $\otimes$ would become to IC (S1) $(\mathrm{M})=\mathrm{M}^{\prime}->\mathrm{IC}(\mathrm{S} 2)\left(\mathrm{M}^{\prime}\right)$, error).

\section{Assuming uncurried and named functions}

First we assume that for any curried function (e.g. for IE and IC of VSPL) a new uncurried version is applied in the sequel. Any application of a curried function has to be substituted by the application of the corresponding uncurried version. Second we can assume the absence of lambda abstractions, since we don't represent functional domains at all.

\subsection{Derivation of correct logic programs}

We are going to describe the derivation of a logic program $\mathrm{SP}=<\mathrm{PRED}, \mathrm{FUNC}, \mathrm{CLAUS}, \mathrm{VAR}>$ from the denotational semantics SD. Therefore a term representation of elements of any domain applied within SD is given. Furthermore the implementation of the functional equations by definite clauses is described. For that purpose a set of sorts SDCP, an SDCP-signature FDCP and an SDCP-sorted set of variables VDCP (all together describing the set of terms which can be applied within SP) will be constructed. We assume, that SP is "well-typed", i.e. any p E PRED can be considered as many-sorted predicate symbol (w.r.t. SDCP), FDCP declares the signatures of the function symbols of FUNC; VAR can be partitioned as in VDCP (which corresponds to the meta-variables of SD) and the terms and atoms of CLAUS are "welltyped". Moreover we need a logical language LSP $=\langle$ SDCP, FSP,RSP,VSP $\rangle$, which will be applied to write down the specification SSP of SP and which is interpreted by an LSP-structure DSP mirroring suitable components of the denotational semantics SD. The components of LSP are defined as follows:

- VSP is an SDCP-sorted set of variables beeing an extension of VDCP and containing the variables from varg(p) for any $p \in \operatorname{PRED}$ (see 3.2, Definition 1).

- FSP is an SDCP-signature beeing an extension of FDCP. Additional function symbols beeing place holders for the functions from $\mathrm{SD}$ are included, where we use as function symbol the same name as for the corresponding function in SD, but quoted, e.g. cond' is a function symbol which is interpreted by the function cond in DSP.

- RSP offers predicate symbols for usual equality $(=)$ for any domain.

The specification SSP of SP will assign to any predicate from SP a formula from LSP $_{S}$ stating that the parameters of the predicate are in relation as defined by the corresponding function from $\mathrm{SD}$.

We will construct an injective function $\phi$ assigning to any domain of SD the corresponding sort from SDCP. For bottom elements of the semantic domains no representation is defined. Such a representation is not needed, since we use semantics definitions which do not apply $\perp$, especially not for error handling. The latter is done by including additional error elements. We introduce the notion $>D<$ for any domain $D$ refering to the set of all. representable values from $D .>D<$ is different from $D$ only for semantic domains, where $>\mathrm{D}<$ contains any element from $\mathrm{D}$, but the bottom element and (for domains of products, sequences and sums) such containing bottom elements. For any domain D we will 
construct a function $\pi \mathrm{D}:>\mathrm{D}<->\mathrm{T}(\mathrm{FDCP}) \phi(\mathrm{D})$ defining the representation of elements of $>D<$ as ground terms of the corresponding sort. For any function symbol $f$ included into FDCP an interpretation within $D_{S P}$ will be defined by $\Psi(f)$.

\section{Syntactical domains}

The representation of elements from syntactical domains is straightforward. A syntactical element, which is a term per definition, is represented within the logic program as the same term. The function symbols of FSyn are interpreted as term constructors by $\Psi$.

\section{Elementary domains}

Elements from finite elementary semantic domains (e.g. BOOLEAN) are represented by constant symbols. Thus we include for any element from a finite elementary domain a constant symbol (with the same notation) into FDCP to be interpreted as the corresponding element in DSP. For infinite elementary semantic domains (e.g. INTEGER, ID) a finite number of function symbols of appropriate signature must be applied allowing the construction of an infinite set of (ground) terms where each term represents one element from the domain, e.g. the natural numbers could be represented as usual by zero: $\rightarrow>$ nat, succ: nat $->$ nat, where zero is interpreted as 0 and succ as successor function. Within a concrete Prolog implementation offered data types can be exploited.

\section{Domains of sequences}

Let be $\mathrm{D}$ an arbitrary domain for which a representation is aiready defined. In order to represent elements from $\mathrm{D}^{*}$ in SP we include a new sort SD* in SDCP and two new function symbols [ ]D and $[\ldots \mid \ldots] \mathrm{D}$ in FDCP (to represent the empty list and nonempty lists resp.), where: $\quad \phi\left(D^{*}\right)=s D^{*}$

$$
\begin{aligned}
& {[] \mathrm{D}:->\mathrm{sD}^{*}} \\
& {[\ldots \mid \ldots] \mathrm{D}: \phi(\mathrm{D}) \times \mathrm{sD}^{*} \rightarrow \mathrm{sD}^{*}}
\end{aligned}
$$

$\pi \mathrm{D}^{*}(\diamond)=\left[\right.$ ]D. Let be $\mathrm{n}>0, \forall \mathrm{d}_{\mathrm{i}} \in \mathrm{D}, \mathrm{i}=1, \ldots, \mathrm{n}$ :

$$
\pi D^{*}\left(<d_{1}, \ldots, d_{n}>\right)=\left[\pi D\left(d_{1}\right) \mid \pi D^{*}\left(<d_{2}, \ldots, d_{n}>\right)\right] D .
$$

The interpretation of []$D$ is the empty list and of $[\ldots \ldots \ldots] D$ is a list constructor, i.e. $\Psi([\mathrm{D})=>$ and $\Psi([\ldots 1 \ldots] \mathrm{D})=$ cons.

Whenever the index $\mathrm{D}$ in $[\mathrm{D}$ and $[\ldots . \ldots] \mathrm{D}$ is uniquely defined by the context we will omit $\mathrm{D}$. The representation of tupels from product domains is similar to that of sequences.

\section{Sum domains}

For every summand of sum domains a unique constructor will be used to build the representation by surrounding the representation of the element from the summand domain. Let $\mathrm{D}_{1}, \ldots, \mathrm{D}_{\mathrm{n}}$ be arbitrary domains for which a representation is already defined, $D=\left(D_{1}+\ldots+D_{n}\right)$. In order to represent elements from $D$ in SP we include a new sort SD in SDCP, new function symbols $f_{1}, \ldots, f_{n}$ in FDCP, where $f_{i} \neq f_{j}$ if $i \neq j$, and a bijective function $\alpha \mathrm{D}:\{1, \ldots, \mathrm{n}\} \rightarrow\left\{\mathrm{f}_{1}, \ldots, \mathrm{f}_{\mathrm{n}}\right\}$ assigning to any summand a certain function symbol, i.e. $\alpha D(i)=f_{i}$, where $i=1, \ldots, n$. Furthermore we define:

$$
\begin{aligned}
& \phi(D)=s D \\
& f_{1}: \phi\left(D_{1}\right)->S D, \ldots, f_{n}: \phi\left(D_{n}\right) \rightarrow s D .
\end{aligned}
$$


Let be $1 \leq \mathrm{i} \leq \mathrm{n}, \forall \mathrm{d}_{\mathrm{i}} \in \mathrm{D}_{\mathrm{i}} ; \pi_{\mathrm{D}}\left(\left(\mathrm{d}_{\mathrm{i}}, \mathrm{i}\right)\right)=\mathrm{f}_{\mathbf{i}}\left(\pi_{\mathrm{D}_{\mathrm{i}}}\left(\mathrm{d}_{\mathrm{i}}\right)\right), \Psi\left(\mathrm{f}_{\mathrm{i}}\right)=\mathrm{in}_{\mathrm{i}}$. To simplify the representation we declare a special case, if $D_{j}$ with $1 \leq j \leq n$ is an elementary semantic domain of the form $\{\omega\}_{\perp}$, then: $\mathrm{f}_{\mathrm{j}}:->\mathrm{sD}_{\mathrm{D}}, \pi_{D}((\omega, \mathrm{j}))=\mathrm{f}_{\mathrm{j}}, \Psi\left(\mathrm{f}_{\mathrm{j}}\right)=\underline{\mathrm{in}}_{\mathrm{j}}(\omega)$.

It can be proved, that for any domain $\mathrm{D} \pi_{\mathrm{D}}$ is a bijective function ([Lä93]), i.e. different elements from a domain $\mathrm{D}$ have different images in $\mathrm{T}(\mathrm{FDCP}) \phi(\mathrm{D})$ and $\pi_{\mathrm{D}}{ }^{-1}$ is defined.

\section{Implementing recursive function definitions}

Let $\mathrm{D}_{1}, \ldots, \mathrm{D}_{\mathrm{k}}, \mathrm{D}_{\mathrm{k}+1}, \ldots, \mathrm{D}_{\mathrm{m}}$ be arbitrary domains, where $\mathrm{m} \geq 1$ and $\mathrm{k}<\mathrm{m}$. If f: $D_{1} \times \ldots \times D_{k} \rightarrow D_{k+1} \times \ldots \times D_{m}$ is the function from $S D$ to be implemented within SP, the predicate $\mathrm{p}$ is considered as a correct implementation of $\mathrm{f}$ if:

$$
\begin{aligned}
& \forall \mathrm{x}_{1} \in>\mathrm{D}_{1}<, \ldots, \forall \mathrm{x}_{\mathrm{m}} \in>\mathrm{D}_{\mathrm{m}}<: \\
& \quad \mathrm{p}\left(\pi_{\mathrm{D} 1}\left(\mathrm{x}_{1}\right), \ldots, \pi_{\mathrm{D}_{\mathrm{m}}}\left(\mathrm{x}_{\mathrm{m}}\right)\right) \in \mathrm{DEN}(\mathrm{SP}) \text { iff } \mathrm{f}\left(\mathrm{x}_{1}, \ldots, \mathrm{x}_{\mathrm{k}}\right)=<\mathrm{x}_{\mathrm{k}+1}, \ldots, \mathrm{x}_{\mathrm{m}}>
\end{aligned}
$$

Since we have not defined representations of the bottom elements of any semantic domain in $\left(^{*}\right)$ only representable assignments are taken into consideration. The predicates are not intended to "process" or "return" bottom elements or elements containing bottom elements. Including bottom elements in our considerations would unnecessarily blow up our implementation. In the sequel we always assume assignments restricted to representable values as explicitly stated in $(*)$. To prove $\left(^{*}\right)$ two steps have to be processed:

$(\Rightarrow)$ Any computed answer (by p) is correct w.r.t. f. Below we consider the implementation of particular functions and prove $(\Leftrightarrow)$ in interconnection using the inductive proof method (see 3.2).

$(<$ ) Any pair of argument and result of $\mathrm{f}$ builds an answer of $\mathrm{p}$. This problem is sketched at the end of this subsection following [DF89] (so-called completeness).

\section{Predicates implementing basic functions}

For some basic functions one could assume ad hoc implementations.

Example 3 (Implementation of is ):

Let be $D=\left(D_{1}+\ldots+D_{n}\right), 1 \leq i \leq n$. The function is is implemented by the predicate pisi described by the following clauses:

$$
\begin{aligned}
& \operatorname{pisi}_{i}\left(f_{i}\left(X_{\mathfrak{i}}\right), \text { true }\right) \text {. } \\
& \text { pisi }\left(f_{j}(X j), \text { false }\right) .
\end{aligned}
$$

where $j=1, \ldots, n$ and $j \neq i$ and $f_{k}=\alpha D(k)$ for $k=1, \ldots, n$. If $f_{k}$ is a constant symbol - as mentioned for the special case of summands built from a set with one element - the first parameter position of $p_{i s i}$ has not the form $f_{k}\left(X_{k}\right)$ but the form $f_{k}$.

This implementation method for is $\mathrm{i}$ will be applied to a particular sum domain and the implementation will be proved to be correct. Let be $\left.\mathrm{D}=(\text { VALUE }+ \text { (error }\}_{\perp}\right)$, $\alpha_{D}(1)=e o k, \alpha_{D}(2)=$ eerror. Then the implementation of $\underline{\text { is } 1}$ (i.e. isVALUE) is defined by the predicate isev:

$$
\begin{aligned}
& \text { isev(eok(VAL),true). } \\
& \text { isev(eerror,false). }
\end{aligned}
$$

The specification $\boldsymbol{S}^{\text {isev }}$ (following the notation as introduced in 3.2) for isev describing the relationship between its second and first parameters is: isev $2=\underline{i s} 1$ '(isev1). To prove the correctness of isev w.r.t. $\boldsymbol{S}^{\text {isev }}$ it remains to show that the following formulae are valid (applying DSP as interpretation):

(I) true $=$ is 1 '(eok(VAL))

(II) false $=$ is $1^{\prime}$ (eerror) 
In $D_{S P}$ eok and eerror will be interpreted as in1 and inz(error) resp. (see definition of $\Psi$ for constructors of elements of sum domains). Concerning (I): is 1 (in 1 (VAL')) = is $1\left(\left(V_{A L}^{\prime}, 1\right)\right)=$ true for all VAL' $\varepsilon>V_{A L U E}<$, thus (I) is valid. Concerning (II): $\underline{\text { is } 1}($ in2 $($ error $))=\underline{\text { is } 1}(($ error, 2$))=$ false and thereby (II) is valid.

\section{Basic functions implemented by term construction}

Basic functions need not always to be implemented by special predicates. For example it is sometimes possible to implement them by term construction on parameter positions where their result is needed, e.g. the representation of the result of an application of $i_{i}$ w.r.t. a sum domain $\mathrm{D}$ is always obtained from the representation of the argument of $\mathrm{in}_{\mathrm{i}}$ by surrounding it with $\propto \mathrm{D}(\mathrm{i})$.

\section{Composition operator}

The most important tool to derive predicates from recursive function definitions is the rule how to handle the composition operator ${ }^{\circ}$. If the function $f$ is defined as a composition $f_{n}{ }^{\circ} \ldots{ }^{\circ} f_{1}$ of the functions $f_{1}, \ldots, f_{n}$ (i.e. $f(x)=f_{n}\left(\ldots\left(f_{1}(x) \ldots\right)\right)$ and $p_{1}, \ldots, p_{n}$ are implementations of $f_{1}, \ldots, f_{n}$ resp. in the sense of $(*)$, then the predicate $p$ defined by the definite clause $p\left(X_{1}, X_{n+1}\right)$ :- $p_{1}\left(X_{1}, X_{2}\right), \ldots, p_{n}\left(X_{n}, X_{n+1}\right)$ is certainly a correct implementation of $f$ in the sense of $\left({ }^{*}\right)$.

\section{Special implementation of conditionals}

For reasons of the implementational behaviour we suggest to figure out every application of cond in an additional predicate as follows. Let be $\mathrm{D}_{1}, \mathrm{D}_{2}$ arbitrary domains, 1,r: $\mathrm{D}_{1} \rightarrow$ $\mathrm{D}_{2}, \mathrm{p}_{1}$ and $\mathrm{p}_{\mathrm{r}}$ are implementations of 1 and $\mathrm{r}$ resp., then $\mathrm{f}$ : BOOLEAN $\times \mathrm{D}_{1} \rightarrow \mathrm{D}_{2}$ with

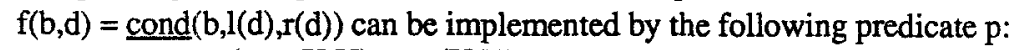

$$
\begin{aligned}
& \mathrm{p}(\text { true, } X, Y):-\operatorname{pl}(X, Y) \text {. } \\
& \mathrm{p}(\text { false }, X, Y):-\operatorname{pr}(X, Y) .
\end{aligned}
$$

Clearly, this implementation is correct. Implementing an $f^{\prime}: D_{1} \rightarrow D_{2}$ with $f^{\prime}(x)=$ cond $\left(e(x), l^{\prime}(x), r^{\prime}(x)\right)$, e: $D_{1} \rightarrow$ BOOLEAN, $l^{\prime}, r^{\prime}: D_{1} \rightarrow D_{2}$, one can often avoid an explicit implementation of $e$ if the result of $e(x)$ (true or false) corresponds to a certain term structure of $\pi \mathrm{D} 1(x)$. The clauses implementing $\mathrm{f}^{\prime}$ can then ensure their applicability by ensuring the certain term structure of the input parameter.

Example 4 (Special handling of cond):

We give an implementation for the auxiliary function APPLY introduced in Example 2. Therefore we define the constructors for the sum domain $D=\left(\right.$ VALUE $\left.+\{\text { undef }\}_{\perp}\right)$ as follows: $\alpha_{D}(1)=$ mok, $\alpha_{D}(2)=$ merror. The predicate apply implements APPLY using the following clauses, where these clauses implement directly the three possible paths through APPLY as controlled by the two applications of cond within APPLY:

(A1) apply( $\square, V$,merror).

(A2) apply([(V,VAL)|M],V,mok(VAL)).

(A3) apply([(V1,VAL)IM],V,MR) :- V1 $\mathrm{l}==\mathrm{V}$, apply(M,V,MR).

As it can be seen for the functions null, head, tail, eli applied to define APPLY no special implementation is required. For the predicates applied in (A1) - (A3) the specifications are: 
sapply : $\quad$ apply3 $=$ APPLY' (apply1,apply2)

st=: $\quad \sim b=1=t=2$

To prove Sapply the validness of the formulae (I) - (III) has to be considered:

(I) merror $=\operatorname{APPLY}^{\prime}([\mathrm{]}, \mathrm{V})$

(II) $\operatorname{mok}(\mathrm{VAL})=\mathrm{APPLY}([(\mathrm{V}, \mathrm{VAL}) \mid \mathrm{M}], \mathrm{V})$

(III) $\quad \sim \mathrm{V} 1=\mathrm{V} \wedge \mathrm{MR}=\mathrm{APPLY}(\mathrm{M}, \mathrm{V}) \Rightarrow \mathrm{MR}=\mathrm{APPLY}([(\mathrm{V} 1, \mathrm{VAL}) \mid \mathrm{M}], \mathrm{V})$

Concerning (I): Obviously valid, since APPLY $\left(<>, V^{\prime}\right)=$ in 2 (undef) for any $V^{\prime} \in>$ ID $<$. Concerning (II): APPLY (cons $\left.\left.\left(<V^{\prime}, V^{\prime} L^{\prime}\right\rangle, M^{\prime}\right), V^{\prime}\right)=\underline{\text { in } 1}\left(V_{A L}\right)$ for all representable $\mathrm{V}^{\prime}, \mathrm{VAL}, \mathrm{M}^{\prime}$, since (see definition of APPLY) null (cons $(. .$.$) ) = false and$ el $1\left(\right.$ head $\left(\right.$ cons $\left.\left.\left(\left\langle V^{\prime}, V A L^{\prime}\right\rangle, M^{\prime}\right)\right)\right)=V^{\prime}$ and el2 $\left(\right.$ head $\left(\right.$ cons $\left.\left.\left(\left\langle V^{\prime}, V^{\prime} L^{\prime}\right\rangle, M^{\prime}\right)\right)\right)=V A L^{\prime}$. Concerning (III): APPLY (cons $\left.\left.\left(<V 1^{\prime}, V A L^{\prime}\right\rangle, M^{\prime}\right), V^{\prime}\right)=\operatorname{APPLY}\left(M^{\prime}, V^{\prime}\right)$ for all representable $V^{\prime}, V^{\prime}, V A L ', M^{\prime}$ with $V^{\prime} \neq V^{\prime}$, since null $($ cons $(. .))=$. false and tail $\left(\right.$ cons $\left.\left.\left(<V 1^{\prime}, V A L^{\prime}\right\rangle, M^{\prime}\right)\right)=M^{\prime}$.

\section{Equations of semantic functions}

Semantic functions are usually described in such a way, that for any alternative for constructing elements from the domain to which meanings are assigned to (i.e. for any function symbol of the considered sort) a separate equation is given. The reflection within the derived logic program is straightforward: for every equation of a semantic function one definite clause has to be included.

Example 5 (Implementing equations of semantic functions):

The semantic function IC will be implemented by the predicate com. E.g. the following two clauses correspond to the semantic clauses (SD.2) and (SD.3).

(C1) $\operatorname{com}($ skip,M,cok(M)).

(C2) $\operatorname{com}($ concat $(\mathrm{S} 1, \mathrm{~S} 2), \mathrm{M}, \mathrm{CR})$ :- $\operatorname{com}(\mathrm{S} 1, \mathrm{M}, \mathrm{CR} 1)$,concat1(S2,CR1,CR).

The application of cond in (SD.3) (changed as above: IC (concat(S1,S2),M) $=$ IC (S1,M) $=\mathrm{M}^{\prime} \rightarrow \mathrm{IC}\left(\mathrm{S} 2, \mathrm{M}^{\prime}\right)$, error) was figured out as described above, where the resulting predicate is concat1. Therefore (C2) implementing (SD.3) refers to concat1 with the following specification:

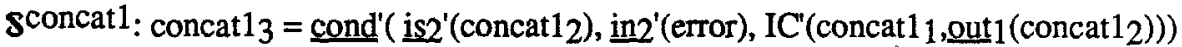

If $\mathrm{D}=\left(\mathrm{MEMORY}+\{\text { error }\}_{\perp}\right), \alpha_{\mathrm{D}}(1)=\mathrm{cok}, \alpha_{\mathrm{D}}(2)=$ cerror, the specification scom: $\operatorname{com}_{3}=\mathrm{IC}^{\prime}\left(\operatorname{com}_{1}, \operatorname{com}_{2}\right)$ can easily be proved to be valid.

Now we want to sketch how to prove $(\Leftrightarrow)$. The main idea for proving that the predicate $\mathrm{p}$ is complete w.r.t. the specification SP (i.e. w.r.t. a certain function) is to ensure that for any possible assignment $v$ satisfying $\mathcal{S P}$ (in our case for any pair of function argument and corresponding function result) it is possible to find an instance of a clause for $p$ such that the parameters in the head instance are equal to $v\left(p_{1}\right), \ldots, v\left(p_{n}\right)$ (starting the "construction") and the parameters in the instances of the atoms in the body are assignments satisfying SSP (ensuring the possibility to continue the "construction"). More roughly spoken, if $p$ is the predicate implementing the function $f$, we have to prove that for any pair of argument 
and result for $f$ there is a clause of $p$ which is applicable on the representations of this pair and where the body implements at least $f$ restricted to this case.

Example 6 (Completeness of apply, see also Example 4):

As specifications of the clauses (A1) - (A3) we take the parts of the definition of APPLY which we intended to implement by the clauses (as demanded by the conditionals).

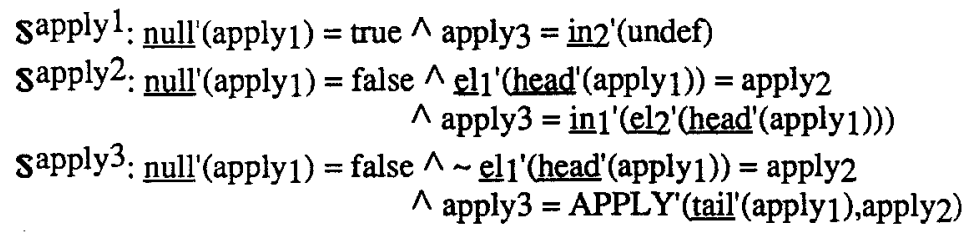

The completeness follows from the construction.

Proving the completeness of predicate p w.r.t. $\mathrm{SP}$ possible "recursive calls" have to be considered to be finite. For denotational functions another property is important, that is to say the "construction" should terminate iff the corresponding computation terminates (e.g. an infinite loop will never terminate and thereby the corresponding recursion could not be proved to be finite). To prove the recursion for a predicate $p$ to be finite, we must give a function $\mu_{p}$ mapping all assignments for parameters of $p$ into a set in which " $<$ " denotes a well-founded relation. During the "construction" as sketched above for any instance $q\left(a^{\prime} 1, \ldots, a^{\prime} m\right)$ in the body of a clause of $p$ which could correspond to a recursive call of $p$ one has to check that $\mu_{\mathrm{q}}\left(\mathrm{a}^{\prime} 1, \ldots, \mathrm{a}_{\mathrm{m}} \mathrm{m}\right)<\mu_{\mathrm{p}}\left(\mathrm{a}_{1}, \ldots, \mathrm{a}_{\mathrm{n}}\right)$, where $\mathrm{p}\left(\mathrm{a}_{1}, \ldots, \mathrm{a}_{\mathrm{n}}\right)$ is the instance of the head.

Example 7 (Proving the finite recursion of apply):

We assume $\mu_{\text {apply }}(\mathrm{M}, \ldots, \ldots)$ as length of list $\mathrm{M}$. There is direct recursion in (A3). Thus we have to check the assignments for $\mathbf{S}^{\text {apply } 3}$. With $<$ as usual for $\mathbb{N}_{0}$, it is observable that $\mu_{\text {apply }}\left(\left[\ldots \mid \pi_{\text {MEMORY }}\left(M^{\prime}\right)\right], \ldots, \ldots\right)<\mu_{\text {apply }}\left(\pi \operatorname{MEMORY}\left(M^{\prime}\right), \ldots, \ldots\right)$.

\section{Conclusions and future work}

We have presented an approach for deriving a provable correct prototype interpreter (as a Prolog program) directly from a language definition consisting of a special kind of attribute grammars and a semantics definition in the denotational style. The described approach is used in the Language Development System (LDL).

The main advantages of our approach are the following (see also the Introduction):

- It contains a method how to derive the Prolog program offering a prototype interpreter from the language definition.

- Our language definitions avoid some disadvantages (e.g. mixing of static and dynamic semantics or unsufficient description of static semantics) of other well-known forms.

- The obtained prototype interpreter can easily be shown to be correct w.r.t. the language definition and can be extended by new components preserving correctness. 
- In difference to us other approaches using Prolog for the implementation of denotational semantics, usually apply the lambda notation and/or execute the semantics description on a lambda machine, which for instance introduces remarkable inefficiency.

There are the following points for further investigation:

- The described implementation method for the denotational semantics should be proved to be semantics preserving in general. Afterwards - assuming a correct implementation of the method itself - prototype interpreters could be derived automatically.

- The approach for proving the correctness of the derived prototype interpreter exploiting the inductive proof method (as applied in this paper) should be compared with the algebraic approach as considered e.g. in [Ma79], [RL93].

- There are other logic programming languages which seem to be more useful as implementation language of the prototype interpreters and for LDL at all, e.g. Gödel with its facilities for meta-programming and modules and its many-sorted type system.

- The operational semantics of our prototype interpreters should be considered more completly and formally. Moreover we are looking for ways to get more realistic prototype interpreters (e.g. as in [L89]).

\section{References}

[AM91] Alblas,H., Melichar,B. (Eds.) : Attribute grammars, Applications and Systems, Proc. of the International Summer School SAGA. Prague, Czechosiowakia, June 1991, LNCS \# 545, Springer-Verlag

[D90] Deransart, P.: Proof Methods of Declarative Properties of Definite Programs, Rapports de Recherche No 1248, INRIA, Juni 1990

[DF89] Deransart, P.; Ferrand, G.: A methodological view of logic programming with negation, In: O.M. Tammepuu (Ed.), Informatics '89, Proc. of the Soviet-French Symposium, Tallinn, May 29 - June 2, 1989, Estonian Academy of Sciences, Institute of Cybernetics, 76 - 91

[K91] Koskimies,K.: Object-orientation in attribute grammars, In: [AM 91], 297-329

[KP92] Kastens,U., Pfahler,P. (Eds.): Compiler Construction, 4th International Conference, CC '92, Paderbom, FRG, Oct. 1992, Proc., LNCS \# 641, Springer-Verlag

[KPy80] Krishnaswamy,R., Pyster,A.B.: On the correctness of semantic-syntax-directed translations, JACM, Vol. 27, No. 2, April 1980, 338-355

[L89] Lee,P.: Realistic compiler generation, The MIT Press 1989

[L393] Lämmel,R.: Prolog-Implementation denotationaler Semantikbeschreibungen, Diplomarbeit, Universităt Rostock, FB Informatik, Jan. 1993

[M73] Morris,F.L.: Advice on structuring compilers and proving them correct, Proc. ACM Symposium on Principles of Programming Languages, Boston, 1973, 144-152

[Ma79] Majster,M.E.: A unified view of semantics, Technical Report 79-394, Cornell University, Ithace, New York, Dep. of Computer Science, 1979

[PRO92] Buth,B. et al.: Provably correct compiler development and implementation, In: [KP92], 141-155

[R91a] Riedewald,G.: Prototyping by using an attribute grammar as a logic program, In: [AM91], 401-437

[R91b] Riedewald,G.: The LDL - Language Development Laboratory, Preprint CS-1-91, Dec. 91, Universităt Rostock, FB Informatik

[R92] Riedewald,G.: The LDL - Language Development Laboratory, In: [KP92], 88-94

[RL93] Riedewald,G.;Lammel,R.: Provable Correctness of Prototype Interpreters in LDL, Preprint CS-9-93, Sept. 93, Universităt Rostock, FB Informatik

[Sch76] Schmeck,H.: Ein algebraischer Ansatz für Kompilerkorrektheitsbeweise, In: Informatik-Fachberichte 1, 1976, Springer-Verlag, 33-42

[St77] Stoy,J.E.: Denotational Semantics: The Scott-Strachey Approach to Programming Language Theory, MrT-Press, 1977

[To90] Tofte,M.: Compiler Generators, EATCS Monographs on Theoretical Computer Science, Vol. 19, Springer-Verlag, 1990

[TWW81] Thatcher,J.W., Wagner,E.G., Wright,J.B.: More on advice on structuring compilers and proving them correct, Theoretical Computer Science 15 (1981), 223-249 